\section{(2) OPEN ACCESS}

\title{
Bile acid diarrhoea: pathophysiology, diagnosis and management
}

\author{
Alexia Farrugia (D) , ${ }^{1,2}$ Ramesh Arasaradnam (i) 2,3
}

\begin{abstract}
${ }^{1}$ Surgery, University Hospitals Coventry and Warwickshire NHS

Trust, Coventry, UK

${ }^{2}$ Divison of Biomedical Sciences, University of Warwick, Warwick Medical School, Coventry, UK

${ }^{3}$ Gastroenterology, University Hospitals of Coventry and Warwickshire NHS Trust, Coventry, UK
\end{abstract}

\section{Correspondence to}

Professor Ramesh Arasaradnam, Gastroenterology, University Hospitals of Coventry and Warwickshire NHS Trust, Coventry CV2 2DX, UK; R Arasaradnam@warwick.ac.uk

Received 18 February 2020 Revised 14 May 2020 Accepted 27 May 2020
Check for updates

(c) Author(s) (or their employer(s)) 2020. Re-use permitted under CC BY-NC. No commercial reuse. See rights and permissions. Published by BMJ.

To cite: Farrugia $A$, Arasaradnam R. Frontline Gastroenterology Epub ahead of print: [please include Day Month Year]. doi:10.1136/ flgastro-2020-101436

\begin{abstract}
The actual incidence of bile acid diarrhoea (BAD) is unknown, however, there is increasing evidence that it is misdiagnosed in up to $30 \%$ with diarrhoea-predominant patients with irritable bowel syndrome. Besides this, it may also occur following cholecystectomy, infectious diarrhoea and pelvic chemoradiotherapy. BAD may result from either hepatic overproduction of bile acids or their malabsorption in the terminal ileum. It can result in symptoms such as bowel frequency, urgency, nocturnal defecation, excessive flatulence, abdominal pain and incontinence of stool. Bile acid synthesis is regulated by negative feedback loops related to the enterohepatic circulation, which are dependent on the farnesoid $X$ receptor and fibroblast growth factor 19. Interruption of these feedback loops is thought to cause bile acid overproduction leading to BAD. This process may occur idiopathically or following a specific trigger such as cholecystectomy. There may also be an interplay with the gut microbiota, which has been reported to be significantly different in patients with severe BAD.

Patients with suspected BAD are investigated in various ways including radionucleotide imaging such as SeHCAT scans (though this is not available worldwide) and blood tests. However, other methods such as bile acid measurement in stool (either spot test or 48 hours samples) and urine tests have been explored. Importantly, delay in diagnosis and treatment of BAD greatly affects patient's quality of life and may double the overall cost of diagnosis.
\end{abstract}

\section{INTRODUCTION}

Bile acid diarrhoea (BAD), sometimes also known as bile acid malabsorption or bile salt malabsorption (though this is not always the correct terminology), can result in symptoms such as bowel frequency, urgency, nocturnal defecation, excessive flatulence, abdominal pain and incontinence of stool. ${ }^{1}$ This phenomenon was first described in 1967 and was initially

\section{Key points}

- Idiopathic bile acid diarrhoea (BAD) is due to overproduction of bile acids (rather than malabsorption).

- The negative feedback loops involved in bile acid synthesis are interrupted in BAD but there is lack of data regarding what causes the interruption.

- There is increasing evidence of an interplay between the gut microbiota, farnesoid $X$ receptor and fibroblast growth factor 19 in BAD.

- Tests for BAD such as SeHCAT are not available worldwide but alternatives include plasma C4 testing and possibly faecal bile acid measurement.

- Idiopathic BAD is due to overproduction of bile acids (rather than malabsorption).

known as choleric enteropathy. ${ }^{2}$ It has since been classified primary BAD which is idiopathic and is usually secondary to hepatic overproduction of bile acids due to interruption of the negative feedback loop rather than malabsorption in the ileum. ${ }^{3}$ Secondary BAD is secondary to a terminal ileum resection or occurs in conditions such as Crohn's disease or postradiation where the terminal ileum reabsorbs bile acids as part of the enterohepatic circulation, thus being a true malabsorption syndrome.

Emerging evidence over the last decade has shown that BAD is not as uncommon as previously perceived. Up to $30 \%$ of patients with diarrhoea-predominant irritable bowel syndrome (IBS) have evidence of BAD as determined by ${ }^{75} \mathrm{SeHCAT}$ testing. ${ }^{4}$ Compared with controls, patients with IBS had lower ${ }^{75} \mathrm{SeHCAT}$ values and higher C4 levels but similar fibroblast growth factor 19 (FGF-19) levels and more than 50\% responded to bile acid sequestrant (colestipol). ${ }^{5}$ In addition to patients with ileal disease (eg, Crohn's disease and right hemicolectomy 
Table 1 Causes of bile acid diarrhoea

\begin{tabular}{ll}
\hline Hepatic overproduction & True malabsorption \\
\hline Idiopathic & Crohn's disease \\
\hline Postcholecystectomy & Right hemicolectomy \\
\hline Irritable bowel syndrome-diarrhoea & Enteropathy (such as HIV (human \\
predominant type & immunodeficiency syndrome) \\
Pancreatic insufficiency & Pelvic radiation \\
& Bariatric surgery \\
& Microscopic colitis \\
& Small bowel bacterial overgrowth \\
\hline
\end{tabular}

where the terminal ileum is resected or HIV causing enteropathy), ${ }^{67}$ BAD has also been reported in those following cholecystectomy, ${ }^{8}$ and those with postinfectious diarrhoea, ${ }^{9}$ as well as patients having metformin ${ }^{10}$ and those with pancreatic insufficiency ${ }^{11}$ (table 1). For those not responding to treatment, other additional causes should be sought, for example, bacterial overgrowth, pancreatic insufficiency or microscopic colitis, ${ }^{12}$ even if ${ }^{75}$ SeHCAT testing has been abnormal. Another under recognised group are those with cancer especially those receiving pelvic chemoradiotherapy as $>50 \%$ have BAD. ${ }^{13}$

Bile acids are vital in the digestion and absorption of fat. They are synthesised from cholesterol in the liver and excreted in bile as primary bile acids (PBA) (cholic and chenodeoxycholic acids). Dehydroxylation then occurs to form lithocolic and deoxycholic acid and these are reabsorbed via the enterohepatic circulation. There is a negative feedback process regulating this, working through the nuclear farnesoid X receptor (FXR) and FGF19. Disruption of this process results in excessive bile acid loss to the colon which, among other factors are contributory to symptoms of diarrhoea.

\section{PATHOPHYSIOLOGY}

\section{Bile acid synthesis and enterohepatic circulation}

Bile acids are synthesised in the liver from cholesterol into PBA (cholic acid (CA) and chenodeoxycholic acid (CDA)) by means of CYP7A1 (cholesterol $7 \alpha$ hydroxylase) enzyme. Some are converted to lithocholic and deoxycholic acid by means of $7 \alpha$ hydroxylase produced by intestinal bacteria and are then expressed into the duodenum to aid in digestion of fatty acids. ${ }^{14}$ The latter is achieved by emulsification and formation of micelles. The diameter of the micelles is $4-5 \mathrm{~nm}$ which allows them pass into the intermicrovillous spaces and reach the epithleial cells to allow for absorption. ${ }^{15}$ Approximately $95 \%$ of bile acids are reabsorbed from the ileum per cycle and the amount of cycles per day tends to vary between individuals, though it is estimated that bile acids actually undergo enterohepatic cycling about five times per day. ${ }^{16} 17$ The cumulative daily loss of bile acids in faeces daily is $200-400 \mathrm{mg}$, however, the total bile acid pool is between 3 and $4 \mathrm{~g} .{ }^{18}$ The enterohepatic cycling process ensures that there are sufficient quantities of bile acids to handle dietary fat. Remarkably this process still remains efficient in most individuals even with the onslaught of high fat within the modern diet. Bile acid production may also be stimulated by dietary intake, such as ingestion of long-chain triglycerides possibly through FXR receptor. ${ }^{1920}$

\section{Negative feedback loops}

Bile acids in the terminal ileum are absorbed via apical sodium-dependent bile acid transporter to activate ileal FXR, which induces transcription of FGF19. This is then released into the portal circulation and travels to the liver to activate hepatic FXR which acts on CYP7A1 via short heterodimer primer, thus decreasing bile acid synthesis. ${ }^{21}$ FGF 19 also binds to FGF receptor 4 (FGFR4) in the hepatocytes, which interacts with $\beta$-klotho (KLB) to inhibit CYP7A1 leading to a further decrease in bile acid synthesis via the classical pathway and activating hepatocyte FXR. ${ }^{22} 23$ Production of FGF19, therefore, inhibits BA synthesis by these two negative feedback loops. ${ }^{24}$ These processes are shown in figure 1.

\section{Bile acid diarrhoea}

The mechanism behind primary BAD relates to the negative feedback mechanism in the rate-limiting step catalysed by CYP7A1. When the negative feedback mechanism is disrupted (potentially due to impaired FGF19 signalling), as occurs in BAD, the activity of CYP7A1 is increased with resultant sixfold to sevenfold increase in the synthesis of bile acids. ${ }^{25}$ Bile acids have been shown to induce fluid secretion and increase mucosal permeability in the colon, cause high amplitude propagated contractions. ${ }^{26-28}$

\section{Putative mechanisms of symptoms in BAD}

Patients with BAD usually present with diarrhoea, which may be persistent or intermittent, frequency, urgency, flatulence, abdominal pain, octurnal defecation and even faecal incontinence. ${ }^{1}$ There is no actual malabsorption in primary $\mathrm{BAD}$, unlike that occurring due to terminal ileal disease such as Crohn's. Rather, there is hepatic overproduction of bile acids due to interruption of the negative feedback loop regulating bile acid synthesis, resulting in a larger than normal proportion of bile acids entering the colon and exerting its effects. ${ }^{3}$ Beyond this the reason behind the development of BAD has not yet been determined, such as where the disruption of this negative feedback loop occurs. There has been a suggestion that there are different phenotypes of $\mathrm{BAD}$, including possible associations in patients with familial hypertriglyceridaemia as well as potential functional genetic variation in the receptors such as FGFR4 and $\beta$-klotho. ${ }^{29}$

It is known that the symptoms of BAD occur mainly due to the entry of a higher concentration of bile acids into the colon. Mekhjian et al proposed the theory of 


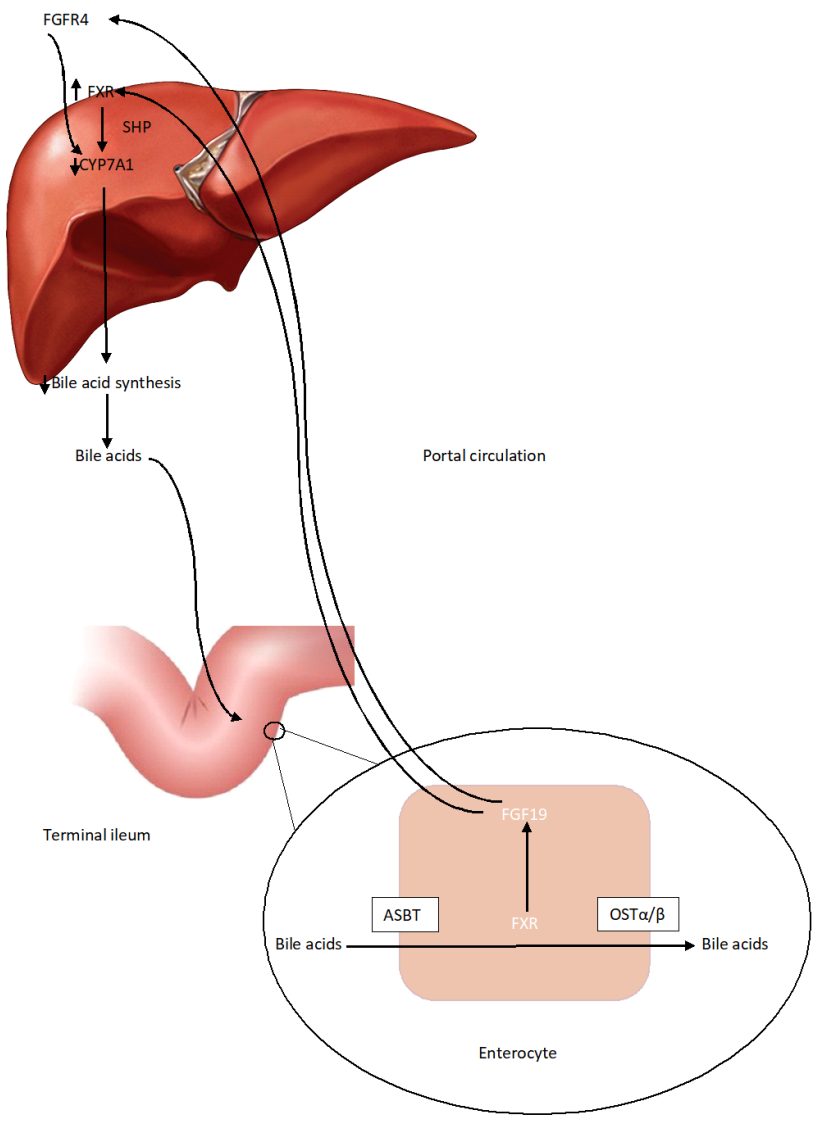

Figure 1 Enterohepatic circulation: Bile acids activate transcription of FGF19 which then acts on hepatic FXR to inhibit bile acid synthesis via CYP7A1. Bile acids are also released into the portal circulation via organic solute transporter (OST) $\alpha / \beta$. They are transported back to the liver where they bind with FGFR4 to interact with $\beta$-klotho to decrease bile acid synthesis via CYP7A1. ASBT, apical sodium-dependent bile acid transporter; FGF19, fibroblast growth factor 19; FGFR4, FGF receptor 4; FXR, farnesoid $X$ receptor; SHP, short heterodimer primer.

induction of intra-luminal secretion of sodium and water. ${ }^{27}$ Others have proposed bile acids stimulating the colonic motility and defecatory reflex, and resultant mucosal damage leading to increased permeability ${ }^{30}$

There have been studies investigating bowel transit and its association with BAD. Some have suggested an accelerated transit both in the small bowel and in the distal colon. ${ }^{31}$ However, other studies suggest no association. ${ }^{32}$ Overall there is little evidence to support claims that symptoms of diarrhoea in BAD are due to changes in intestinal transit, except perhaps for a genetic variation associated with TGR5, a g-protien coupled receptor, (acting on intestinal motility) which increases transit times. ${ }^{33}$

\section{Gut microbiome}

Gut microbiota affect bile acids by causing deconjugation, dehydrogenation and dihydroxylation of PBA in the distal small intestine and colon. This process causes a change in the bile acid pool composition therefore activating FXR and thus inhibiting bile acid synthesis.
However, it is not known whether a change in the gut microbiota has any effect on symptoms of diarrhoea. The amount of secondary bile acids (mainly DCA) in the bile acid pool depends on the rate of formation and absorption via the colon, the colonic transit time and colonic $\mathrm{pH}$. There has been a correlation between high DCA levels and gallstones. ${ }^{34}$

Gut microbiome is involved in the digestion of complex carbohydrates to form short chain fatty acids (SCFA), such as acetate, butyrate and proprionate. ${ }^{35}$ As both the levels of faecal bile acids in the colon and levels of SCFA are dependent on gut microbiota, any changes in faecal bile acids may affect SCFA and in turn effect the presence of diarrhoea. Patients with BAD also have a higher proportion of primary faecal bile acids, potentially due to decreased Bifidobacteria and Leptum species as well as an increased Escherichia coli in their gut microbiota. This may change the affinity of BAs to FXR and TGR5, thus leading to decreased FXR activation and to increased delivery of bile acids to the colon. ${ }^{36}$ Wang et $a l,{ }^{37}$ have shown an increase in Bifidobacteria abundence with concurrent decrease in secondary faecal bile acids and resultant increase in acetate and proprionate levels. The relationship between the increase of SCFA with the decrease in total faecal bile acids has not been explored. The gut microbiome is also heavily involved in the lipid metabolism and absorption, and thus, dysbiosis may affect the interplay between lipids and bile acid synthesis. ${ }^{38}$

There have also been studies investigating the differences in faecal microbiota of different gut conditions. The faecal microbiota of patients with severe BAD $(<5 \%)$ is significantly different to that of patients with diarrhoea secondary to IBS and that of patients with less severe forms of BAD. ${ }^{39-41}$

\section{DIAGNOSTIC METHODS}

There are several methods by which BAD can be diagnosed, all of varying reliability outlined in table 2 .

\section{Nuclear medicine}

The BSG guidelines state that patients with chronic diarrhoea should all be investigated to exclude BAD either with a ${ }^{75} \mathrm{SeHCAT}$ scan where available or $\mathrm{C} 4$ given current evidence base. ${ }^{42}{ }^{75}$ SeHCAT (Selenium-75 homocholic acid taurine test), first described in 1982, is used to determine the amount of bile acids retained after 7 days. ${ }^{43}$ The National Institute for Health and Care Excellence diagnostic guidance report on ${ }^{75} \mathrm{SeHCAT}$ in 2012 stated that given the prevalence of undiagnosed $\mathrm{BAD}$, there is potential for patient and system benefits associated with ${ }^{75} \mathrm{SeHCAT}$ investigations. ${ }^{45}$ The report also suggested that insufficient evidence exists to determine its cost-effectiveness and recommended further research to evaluate this technology and effects of treatment ${ }^{46}$ Its 2016 review, made no changes in light of lack of new evidence on ${ }^{75}$ SeHCATs comparative diagnostic accuracy and 
Table 2 Comparison of diagnostic methods of bile acid diarrhoea (BAD)

\begin{tabular}{lll}
\hline Diagnostic method & Favourable points & Limitations \\
\hline${ }^{75}$ SeHCAT & $\begin{array}{l}\text { Well established } \\
\text { Predicts response to treatment }\end{array}$ & $\begin{array}{l}\text { Involves radiation } \\
\text { Limited availability in certain countries for example, } \\
\text { unavailable in USA }\end{array}$ \\
\hline C4 & $\begin{array}{l}\text { No radiation } \\
\text { Simple }\end{array}$ & $\begin{array}{l}\text { Diurnal variation } \\
\text { Fasting sample }\end{array}$ \\
& No radiation & Not widely available (in the UK as a research tool)
\end{tabular}

FGF19, fibroblast growth factor 19 .

2020 review is awaited. A systematic review and metaanalysis comprising 36 studies and 5028 patients on BAD biomarkers concluded that ${ }^{75} \mathrm{SeHCAT}$ had a highest diagnostic yield to date (limited by study heterogeneity) with $25 \%$ previously diagnosed as having functional bowel disorders actually had primary $\mathrm{BAD}^{47}$

Selenium-75 homocholic acid taurine is a synthetic analogue of taurocholic acid, which is a natural conjugated bile acid, and behaves in the same way as bile acids except that it is resistant to deconjugation by intestinal bacteria. ${ }^{48}$ It is ingested in the form of a capsule after an overnight fast, and a standard gamma camera is used to detect the baseline level 3 hours after ingestion. The scan is repeated after 7 days and the overall retention in the abdomen is measured. Retention values of $10 \%-15 \%$ are considered mild bile acid malabsorption, while $5 \%-10 \%$ is moderate bile acid malabsorption and less than $5 \%$ is severe bile acid malabsorption. ${ }^{45} 49$ However, its use is not widespread despite the ability to be used in any nuclear medicine department supporting a gamma camera and it is not licensed for use in the USA. ${ }^{50}$ Sensitivity of ${ }^{75}$ SeHCAT testing is $96 \%$ with a specificity of $100 \%$ at 7 days. ${ }^{51}$

${ }^{75} \mathrm{SeHCAT}$ may also predict response to therapy. Pooled data from 15 studies shows a dose-response relationship between the severity of malabsorption and the effect of treatment with a bile acid sequestrant: clinical response to colestyramine occurred in $96 \%$ of patients with $<5 \%$ retention of SeHCAT, $80 \%$ at $<10 \%$ retention and $70 \%$ at $<15 \%$ retention. ${ }^{52}$ In general, the lower the SeHCAT retention value the greater the likelihood of response to sequestrants.

\section{Blood}

C4 (7 $\alpha$-hydroxy-4-cholesten-3-one) levels may also be used to diagnose BAD, especially in the absence of ${ }^{75}$ SeHCAT. This is a marker of bile acid synthesis via CYP7A1, and baseline C4 levels are raised in patients with BAD secondary to impaired feedback of FGF19. ${ }^{3}$ This indicates increased bile acid synthesis and thus increased levels of bile acids in the colonic lumen. ${ }^{48}$ Patients with terminal ileal disease may also have increased C4 levels, as decreased reabsorption may increase synthesis. ${ }^{53}$ When compared with ${ }^{75}$ SeHCAT testing, C4 levels have a negative predictive value of $98 \%$, making it an attractive test to exclude BAD. ${ }^{48}$ When comparing ${ }^{75} \mathrm{SeHCAT}$ values $<10 \%$, fasting C4 levels $>48.4 \mathrm{ng} / \mathrm{mL}$ has a sensitivity of $90 \%$ and a specificity of $79 \%{ }^{54}$ Timing of specimen collection is critical due to the diurnal variation of C4 levels as well as variation with hypertriglyceridaemia and ethanol levels (both of which are associated with higher bile acid synthesis, therefore, higher C4 levels). ${ }^{53}{ }^{55-58} \mathrm{C} 4$ levels correlate negatively with faecal bile acid excretion $^{59}$ and inversely correlated with FGF19 levels. ${ }^{60}$

Fasting serum FGF19 levels are inversely correlated with C4 levels. C4 levels are usually higher in patients with BAD. Since FGF19 inhibits bile acid synthesis, decreased FGF19 levels may indicate BAD. FGF19 levels correlate well with ${ }^{75} \mathrm{SeHCAT}$ results, with a negative predictive value of $82 \%$ for ${ }^{75} \mathrm{SeHCAT}$ of $<10 \%$, sensitivity of $58 \%$ and specificity of $84 \%$ for a serum FGF19 level of $<145 \mathrm{pg} / \mathrm{mL}$. Its negative predictive value rises to $94 \%$ for ${ }^{75} \mathrm{SeHCAT}<5 \%,{ }^{60}$ FGF19 levels change rapidly after meals, and there is also a natural diurnal variation. Thus like C4, standardisation would be required if FGF19 levels is to be used for 
diagnosis. ${ }^{61}$ FGF19 levels are not yet routinely used in the diagnosis of BAD.

\section{Stool}

Measurement of faecal bile acids may also be another diagnostic test for BAD if ${ }^{75} \mathrm{SeHCAT}$ is unavailable. ${ }^{62}$ This is a measure of the total excess bile acids exiting the colon. Within the colon, a proportion of bile acids are absorbed and conjugated into secondary bile acids. The amount of primary faecal bile acids (CDA and CA) are found to be higher in patients with BAD and even correlate with frequency and consistency of stool. ${ }^{59} 63$ Patients with BAD have a higher stool weight. Total faecal bile acids of more than $2337 \mu \mathrm{mol} / 48$ hours are diagnostic for $\mathrm{BAD}$, however, elevated primary faecal bile acids may also be used as a diagnostic test, as $>4 \% \mathrm{PBA}$ are indicative of $\mathrm{BAD}$ since healthy volunteers usually only have about $0.02 \%$ primary faecal bile acids. A 4\% cut-off may be used even when total faecal bile acids measure $1000 \mu \mathrm{mol} / 48$ hours. ${ }^{17}$ However, this requires a 48-hour faecal collection taken during the last 2 days of a 4-day $100 \mathrm{~g}$ fat diet, due to variation in dietary fat intake and consequently bile acid levels. Faeces needs to be homogenised, deconjugated and separated before performing either gas chromatography-mass spectrometry, liquid chromatography-tandem mass spectrometry and HPLC-mass spectrometry. ${ }^{64} 65$ This poses difficulty in patient compliance, labour-intensive analytical process and further complicated by the fact that there will also be diurnal variation in bile acid secretion. A pilot study has shown feasibility with a single spot faecal sample but requires further validation, as $>10 \%$ PBA in a faecal spot sample had a $45 \%$ sensitivity and an $63 \%$ to detect ${ }^{75}$ SEHCAT value of $<15 \% .{ }^{66}$ Given that stool uptake in the UK bowel cancer screening programme is $50 \%-58 \% .{ }^{67}$ It is hard to conceive this will be used routinely for diagnosis in the UK due to poor patient compliance coupled with dietary restriction.

\section{Urine}

There has also been a study to detect volatile organic compounds in urine of patients with BAD. This revealed detection of 2-propanol and acetamide as markers of $\mathrm{BAD}$, compared with healthy controls and patients with inflammatory bowel disease. ${ }^{68}$ This may prove a novel way to diagnose BAD but further validation studies are required.

There has also been some experimental work using an electronic nose to detect volatile organic compounds. This is still being investigated, however, the chemical signature profile of patients with BAD were different to those with ulcerative colitis and healthy controls. Its purported mechanisms include gut dysbiosis or dysfermention in response to disease. ${ }^{69}$ The main gases identified in patients with BAD were 2-propanolol and acetamide. ${ }^{68}$

\section{Therapeutic trials}

Bile acid sequestrants are sometimes used in a therapeutic trial if no other diagnostic methods are available. In a series of 264 patients where $53 \%$ had BAD, $44 \%$ failed to respond to cholestyramine alone. Half of these non-responders derived benefit from Colesevelam (unlicensed but used with extended indication). Thus, lack of response to cholestyramine does not constitute exclusion of BAD hence therapeutic trials of bile acid sequestrants (cholestyramine or colesevelam) are not recommended. ${ }^{70}$ This has been affirmed also by the BSG chronic diarrhoea guidelines. ${ }^{42}$ A patient-reported outcome study has shown that if the diagnosis of BAD is delayed, often by more than 5 years, due to poor recognition of the disease patients quality of life is affected negatively. ${ }^{71}$ Delayed investigation and treatment of BAD has been shown to almost double the diagnostic care-of-package cost. ${ }^{72}$

\section{MANAGEMENT}

Bile acid sequestrants are the first-line management of BAD. Colestyramine has long been used as a first line and response has been associated with ${ }^{75} \mathrm{SeHCAT}$ value. Ninety-six per cent of patients with $<5 \%$ retention responded to cholestyramine, $80 \%$ of patients responded at $<10 \%$ retention and $70 \%$ at $<15 \%$ retention. ${ }^{52}$ Colestipol has similarly been shown to improve symptoms of diarrhoea in patients with low ${ }^{75}$ SEHCAT. ${ }^{5}$

Unfortunately, colestryamine and colestipol are often discontinued by patients as they are poorly tolerated due to the taste and texteure of the resin powder. Another issue arises as patients find that while diarrhoea settles, they often have constipation, bloating, nausea and abdominal cramps. ${ }^{73}$ Colesevelam is another bile acid sequestrant which is often better tolerated than colestyramine and has also been shown to create a firmer consistency of stool in such patients, however, its use is unlicenced. ${ }^{74-76}$

Some other medications have been tried, such as hydroxypropyl cellulose which was compared with colestryamine. There was no difference between patients on colestyramine and on hydroxypropyl cellulose who achieved clinical remission in 8 weeks, however, it was found that colestyramine was superior in decreasing the number of watery stools. ${ }^{77}$ Obeticholic acid has been shown to stimulate FGF19, thus reducing bile acid synthesis and causing symptom improvement related to stool frequency and stool form. It was also effective in patients with ileal resections, improving abdominal pain and urgency, though more so in patients with a shorter resected length. ${ }^{78}$

Dietary interventions may be used to improve symptoms. A low-fat diet has been shown to improve gastrointestinal symptoms in patients, with improvements shown in urgency, bloating, lack of control, bowel frequency, abdominal pain and nocturnal defecation. ${ }^{79}$ A combined approach using both 
colesevelam and low fat approach has also been shown to be helpful. ${ }^{81}$

Specific conditions causing BAD secondary to malabsorption will need treatment, such as antibiotics for small bowel overgrowth, ${ }^{82}$ or steroids (mainly budesonide due to lack of systemic side effects) for microscopic colitis. ${ }^{83}$

\section{CONCLUSION}

While part of the pathophysiology behind BAD has been elicited, there are gaps in knowledge as to what causes the disruption of the feedback loop in the case of idiopathic BAD. This may be limiting advances in diagnosis and treatment of the disease. ${ }^{75} \mathrm{SeHCAT}$ or C4 measuement is still the most commonly used methods for diagnosis though novel technologies such as e-nose are emerging. Spot faecal bile acid measurement may hold some potential but requires further validation and may prove difficult to use routinely in clinic. BAD is a treatable disease, however, the delay in diagnosis causes a significant increase in the diagnostic care cost as well as affecting the quality of life of patients. Further avenues for research should look into further defining the reasons behind the disruption of the negative feedback loops to target treatment.

Contributors AF performed the initial literature review and draft of the article. RA reviewed the article and made corrections, suggested entry of further information and received the initial invitation to submit the article.

Funding The authors have not declared a specific grant for this research from any funding agency in the public, commercial or not-for-profit sectors.

Competing interests None declared.

Patient consent for publication Not required.

Provenance and peer review Not commissioned; externally peer reviewed.

Open access This is an open access article distributed in accordance with the Creative Commons Attribution Non Commercial (CC BY-NC 4.0) license, which permits others to distribute, remix, adapt, build upon this work noncommercially, and license their derivative works on different terms, provided the original work is properly cited, appropriate credit is given, any changes made indicated, and the use is noncommercial. See: http://creativecommons.org/licenses/by-nc/4. $0 /$.

\section{ORCID iDs}

Alexia Farrugia http://orcid.org/0000-0002-4366-9068

Ramesh Arasaradnam http://orcid.org/0000-0002-2231-3062

\section{REFERENCES}

1 Walters JRF, Arasaradnam R, Andreyev HJN, et al. Diagnosis and management of bile acid diarrhoea: a survey of UK expert opinion and practice. Frontline Gastroenterol 2020;11:358-63.

2 Hofmann AF. The syndrome of ileal disease and the broken enterohepatic circulation: cholerheic enteropathy. Gastroenterology 1967;52:752-7.

3 Walters JRF, Tasleem AM, Omer OS, et al. A new mechanism for bile acid diarrhea: defective feedback inhibition of bile acid biosynthesis. Clin Gastroenterol Hepatol 2009;7:1189-94.

4 Kurien M, Evans KE, Leeds JS, et al. Bile acid malabsorption: an under-investigated differential diagnosis in patients presenting with diarrhea predominant irritable bowel syndrome type symptoms. Scand J Gastroenterol 2011;46:818-22.

5 Bajor A, Törnblom H, Rudling M, et al. Increased colonic bile acid exposure: a relevant factor for symptoms and treatment in IBS. Gut 2015;64:84-92.

6 Nyhlin H, Merrick MV, Eastwood MA. Bile acid malabsorption in Crohn's disease and indications for its assessment using SeHCAT. Gut 1994;35:90-3.

7 Bjarnason I, Sharpstone DR, Francis N, et al. Intestinal inflammation, ileal structure and function in HIV. AIDS 1996;10:1385-91.

8 Sciarretta G, Furno A, Mazzoni M, et al. Post-Cholecystectomy diarrhea: evidence of bile acid malabsorption assessed by SeHCAT test. Am J Gastroenterol 1992;87:1852-4.

9 Niaz SK, Sandrasegaran K, Renny FH, et al. Postinfective diarrhoea and bile acid malabsorption. J R Coll Physicians Lond 1997;31:53-6.

10 Camilleri M. Bile acid diarrhea: prevalence, pathogenesis, and therapy. Gut Liver 2015;9:332-9.

11 Walters JRF, Pattni SS. Managing bile acid diarrhoea. Therap Adv Gastroenterol 2010;3:349-57.

12 Fernandez-Bañares F, Esteve M, Salas A, et al. Bile acid malabsorption in microscopic colitis and in previously unexplained functional chronic diarrhea. Dig Dis Sci 2001;46:2231-8.

13 Phillips F, Muls ACG, Lalji A, et al. Are bile acid malabsorption and bile acid diarrhoea important causes of loose stool complicating cancer therapy? Colorectal Dis 2015;17:730-4.

14 Takikawa H. Metabolism of Bile Acids. In: Tazuma S, Takikawa $\mathrm{H}$, eds. Bile acids in gastroenterology: basic and clinical. Tokyo: Springer Japan, 2017: 3-8.

15 Honda A, Ikegami T, Matsuzaki Y. Intestinal Digestion and Absorption. In: Tazuma S, Takikawa H, eds. Bile acids in gastroenterology: basic and clinical. Tokyo: Springer Japan, 2017: 27-41.

16 Martinez-Augustin O, Sanchez de Medina F. Intestinal bile acid physiology and pathophysiology. World J Gastroenterol 2008;14:5630-40.

17 Vijayvargiya P, Camilleri M. Current practice in the diagnosis of bile acid diarrhea. Gastroenterology 2019;156:1233-8.

18 Harrison's Principles of Internal Medicine14th ed1998McGraw Hill

19 Ladas SD, Isaacs PE, Murphy GM, et al. Comparison of the effects of medium and long chain triglyceride containing liquid meals on gall bladder and small intestinal function in normal man. Gut 1984;25:405-11.

20 Sagar NM, McFarlane M, Nwokolo C, Bardhan KD, et al. Mechanisms of triglyceride metabolism in patients with bile acid diarrhea. World J Gastroenterol 2016;22:6757-63.

21 Zhou H, Hylemon PB. Bile acids are nutrient signaling hormones. Steroids 2014;86:62-8.

22 Walters JRF. Bile acid diarrhoea and FGF19: new views on diagnosis, pathogenesis and therapy. Nat Rev Gastroenterol Hepatol 2014;11:426-34.

23 Keely SJ, Walters JRF. The farnesoid X receptor: good for bad. Cell Mol Gastroenterol Hepatol 2016;2:725-32.

24 Amigo L, Husche C, Zanlungo S, et al. Cholecystectomy increases hepatic triglyceride content and very-low-density lipoproteins production in mice. Liver Int 2011;31:52-64.

25 Tazuma STHSe T, He T, eds. Bile acids in gastroenterology : basic and clinical. editors: Springer, 2017.

26 Chadwick VS, Gaginella TS, Carlson GL, et al. Effect of molecular structure on bile acid-induced alterations in absorptive function, permeability, and morphology in the perfused rabbit colon. J Lab Clin Med 1979;94:661-74.

27 Mekhjian HS, Phillips SF, Hofmann AF. Colonic secretion of water and electrolytes induced by bile acids: perfusion studies in man. Journal of Clinical Investigation 1971;50:1569-77. 
28 Bampton PA, Dinning PG, Kennedy ML, et al. The proximal colonic motor response to rectal mechanical and chemical stimulation. Am J Physiol Gastrointest Liver Physiol 2002;282:G443-9.

29 Johnston IM, Nolan JD, Pattni SS, et al. Characterizing factors associated with differences in FGF19 blood levels and synthesis in patients with primary bile acid diarrhea. Am J Gastroenterol 2016;111:423-32.

30 McJunkin B, Fromm H, Sarva RP, et al. Factors in the mechanism of diarrhea in bile acid malabsorption: fecal $\mathrm{pH}$--a key determinant. Gastroenterology 1981;80:1454-64.

31 Sadik R, Abrahamsson H, Ung K-A, et al. Accelerated regional bowel transit and overweight shown in idiopathic bile acid malabsorption. Am J Gastroenterol 2004;99:711-8.

32 Sciarretta G, Fagioli G, Furno A, et al. 75Se HCAT test in the detection of bile acid malabsorption in functional diarrhoea and its correlation with small bowel transit. Gut 1987;28:970-5.

33 Camilleri M, Vazquez-Roque MI, Carlson P, et al. Association of bile acid receptor TGR5 variation and transit in health and lower functional gastrointestinal disorders. Neurogastroenterol Motil 2011;23:995-e458.

34 Sagar NM, Cree IA, Covington JA, et al. The interplay of the gut microbiome, bile acids, and volatile organic compounds. Gastroenterol Res Pract 2015;2015:1-6.

35 Soldavini J, Kaunitz JD. Pathobiology and potential therapeutic value of intestinal short-chain fatty acids in gut inflammation and obesity. Dig Dis Sci 2013;58:2756-66.

36 Sayin SI, Wahlström A, Felin J, et al. Gut microbiota regulates bile acid metabolism by reducing the levels of tauro-betamuricholic acid, a naturally occurring FXR antagonist. Cell Metab 2013;17:225-35.

37 Wang L, Zhang J, Guo Z, et al. Effect of oral consumption of probiotic Lactobacillus planatarum P-8 on fecal microbiota, sIgA, SCFAs, and TBAs of adults of different ages. Nutrition 2014;30:776-83.

38 Schoeler M, Caesar R. Dietary lipids, gut microbiota and lipid metabolism. Rev Endocr Metab Disord 2019;20:461-72.

39 Jeffery IB, Das A, O'Herlihy E, et al. Differences in fecal Microbiomes and metabolomes of people with vs without irritable bowel syndrome and bile acid malabsorption. Gastroenterology 2020;158:1016-28.

40 Liu Y, Zhang L, Wang X, et al. Similar fecal microbiota signatures in patients with diarrhea-predominant irritable bowel syndrome and patients with depression. Clin Gastroenterol Hepatol 2016;14:1602-11.

41 Sagar N, Duboc H, Kay G, et al. OWE-021 Describing the gut microbiome and metabolomic changes in bile acid diarrhoea. Gut 2018;67.

42 Arasaradnam RP, Brown S, Forbes A, et al. Guidelines for the investigation of chronic diarrhoea in adults: British Society of gastroenterology, 3rd edition. Gut 2018;67:1380-99.

43 Merrick MV, Eastwood MA, Anderson JR, et al. Enterohepatic circulation in man of a gamma-emitting bile-acid conjugate, 23-selena-25-homotaurocholic acid (SeHCAT). J Nucl Med 1982;23:126-30

44 Merrick MV, Eastwood MA, Ford MJ. Is bile acid malabsorption underdiagnosed? an evaluation of accuracy of diagnosis by measurement of SeHCAT retention. Br Med J 1985;290:665-8.

45 NICE. SeHCAT (tauroselcholic [75 selenium] acid) for the investigation of diarrhoea due to bile acid malabsorption in people with diarrhoea-predominant irritable bowel syndrome (IBS-D) or Crohn's disease without ileal resection. DG7; 2012.

46 Riemsma R, Al M, Corro Ramos I, et al. SeHCAT [tauroselcholic (selenium-75) acid] for the investigation of bile acid malabsorption and measurement of bile acid pool loss: a systematic review and cost-effectiveness analysis. Health Technol Assess 2013;17:1-236.
47 Valentin N, Camilleri M, Altayar O, et al. Biomarkers for bile acid diarrhoea in functional bowel disorder with diarrhoea: a systematic review and meta-analysis. Gut 2016;65:1951-9.

48 Eusufzai S, Axelson M, Angelin B, et al. Serum 7 alphahydroxy-4-cholesten-3-one concentrations in the evaluation of bile acid malabsorption in patients with diarrhoea: correlation to SeHCAT test. Gut 1993;34:698-701.

49 Arasaradnam RP, Cullis J, Nwokolo C, et al. Bile acid malabsorption and SeHCAT: the 'Cinderella' will be going to the Nuclear Medicine Ball! Nucl Med Commun 2012;33:449-51.

50 Smith MJ, Perkins AC. A survey of the clinical use of SeHCAT in the UK. Nucl Med Commun 2013;34:306-13.

51 Sciarretta G, Vicini G, Fagioli G, et al. Use of 23-selena25 -homocholyltaurine to detect bile acid malabsorption in patients with illeal dysfunction or diarrhea. Gastroenterology 1986;91:1-9.

52 Wedlake L, A'Hern R, Russell D, et al. Systematic review: the prevalence of idiopathic bile acid malabsorption as diagnosed by SeHCAT scanning in patients with diarrhoeapredominant irritable bowel syndrome. Aliment Pharmacol Ther 2009;30:707-17.

53 Brydon WG, Culbert P, Kingstone K, et al. An evaluation of the use of serum 7-alpha-hydroxycholestenone as a diagnostic test of bile acid malabsorption causing watery diarrhea. Can J Gastroenterol 2011;25:319-23.

54 Sauter GH, Moussavian AC, Meyer G, et al. Bowel habits and bile acid malabsorption in the months after cholecystectomy. Am J Gastroenterol 2002;97:1732-5.

55 Gälman C, Angelin B, Rudling M. Bile acid synthesis in humans has a rapid diurnal variation that is asynchronous with cholesterol synthesis. Gastroenterology 2005;129:1445-53.

56 Axelson M, Mörk B, Sjövall J. Ethanol has an acute effect on bile acid biosynthesis in man. FEBS Lett 1991;281:155-9.

57 Duane WC. Abnormal bile acid absorption in familial hypertriglyceridemia. J Lipid Res 1995;36:96-107.

58 Camilleri M, Busciglio I, Acosta A, et al. Effect of increased bile acid synthesis or fecal excretion in irritable bowel syndrome-diarrhea. Am J Gastroenterol 2014;109:1621-30.

59 Wong BS, Camilleri M, Carlson P, et al. Increased bile acid biosynthesis is associated with irritable bowel syndrome with diarrhea. Clin Gastroenterol Hepatol 2012;10:1009-15.

60 Pattni SS, Brydon WG, Dew T, et al. Fibroblast growth factor 19 in patients with bile acid diarrhoea: a prospective comparison of FGF19 serum assay and SeHCAT retention. Aliment Pharmacol Ther 2013;38:967-76.

61 Lundåsen T, Gälman C, Angelin B, et al. Circulating intestinal fibroblast growth factor 19 has a pronounced diurnal variation and modulates hepatic bile acid synthesis in man. J Intern Med 2006;260:530-6.

62 Chandrapalan S, Arasaradnam RP. The role of fecal markers in the investigation of patients with chronic diarrhea. Pol Arch Intern Med 2019;129:408-13.

63 Shin A, Camilleri M, Vijayvargiya P, et al. Bowel functions, fecal unconjugated primary and secondary bile acids, and colonic transit in patients with irritable bowel syndrome. Clin Gastroenterol Hepatol 2013;11:1270-5.

64 Griffiths WJ, Sjövall J. Bile acids: analysis in biological fluids and tissues. J Lipid Res 2010;51:23-41.

65 Mitchell WD, Findlay JM, Prescott RJ, et al. Bile acids in the diarrhoea of ileal resection. Gut 1973;14:348-53.

66 Walters JR, Sagar N, Duboc H, et al. Mo1542 - primary bile acids in a single fecal sample for the diagnosis of bile acid diarrhea: relationship to Sehcat testing. Gastroenterology 2019;156:S-774-774.

67 Cancer Research UK. Bowel cancer diagnosis and treatment statistics, 2019. Available: https://www.cancerresearchuk.org/ health-professional/cancer-statistics/statistics-by-cancer-type/ bowel-cancer/diagnosis-and-treatment 
68 Covington JA, Westenbrink EW, Ouaret N, et al. Application of a novel tool for diagnosing bile acid diarrhoea. Sensors 2013;13:11899-912.

69 Chandrapalan S, Arasaradnam RP. Urine as a biological modality for colorectal cancer detection. Expert Rev Mol Diagn 2020;20:489-96.

70 Orekoya O, McLaughlin J, Leitao E, et al. Quantifying bile acid malabsorption helps predict response and tailor sequestrant therapy. Clin Med 2015;15:252-7.

71 Bannaga A, Kelman L, O'Connor M, et al. How bad is bile acid diarrhoea: an online survey of patient-reported symptoms and outcomes. BMJ Open Gastroenterol 2017;4:e000116.

72 Fernandes DCR, Poon D, White LL, et al. What is the cost of delayed diagnosis of bile acid malabsorption and bile acid diarrhoea? Frontline Gastroenterol 2019;10:72-6.

73 Rössel P, Sortsøe Jensen H, Qvist P, et al. Prognosis of adultonset idiopathic bile acid malabsorption. Scand J Gastroenterol 1999;34:587-90.

74 Odunsi-Shiyanbade ST, Camilleri M, McKinzie S, et al. Effects of chenodeoxycholate and a bile acid sequestrant, colesevelam, on intestinal transit and bowel function. Clin Gastroenterol Hepatol 2010;8:159-65.

75 Beigel F, Teich N, Howaldt S, et al. Colesevelam for the treatment of bile acid malabsorption-associated diarrhea in patients with Crohn's disease: a randomized, double-blind, placebo-controlled study. Journal of Crohn's and Colitis 2014;8:1471-9.

76 Camilleri M, Acosta A, Busciglio I, et al. Effect of colesevelam on faecal bile acids and bowel functions in diarrhoea- predominant irritable bowel syndrome. Aliment Pharmacol Ther 2015;41:438-48.

77 Fernández-Bañares F, Rosinach M, Piqueras M, et al. Randomised clinical trial: colestyramine vs. hydroxypropyl cellulose in patients with functional chronic watery diarrhoea. Aliment Pharmacol Ther 2015;41:1132-40.

78 Walters JRF, Johnston IM, Nolan JD, et al. The response of patients with bile acid diarrhoea to the farnesoid X receptor agonist obeticholic acid. Aliment Pharmacol Ther 2015;41:54-64.

79 Watson L, Lalji A, Bodla S, et al. Management of bile acid malabsorption using low-fat dietary interventions: a useful strategy applicable to some patients with diarrhoeapredominant irritable bowel syndrome? Clin Med $2015 ; 15: 536-40$.

80 Jackson A, Lalji A, Kabir M, et al. The efficacy of a low-fat diet to manage the symptoms of bile acid malabsorption outcomes in patients previously treated for cancer. Clin Med 2017;17:412-8.

81 Gupta A, Muls AC, Lalji A, et al. Outcomes from treating bile acid malabsorption using a multidisciplinary approach. Support Care Cancer 2015;23:2881-90.

82 Roberts SH, James O, Jarvis EH. Bacterial overgrowth syndrome without "blind loop": A cause for malnutrition in the elderly. Lancet 1977;2:1193-5.

83 Bajor A, Kilander A, Gälman C, et al. Budesonide treatment is associated with increased bile acid absorption in collagenous colitis. Aliment Pharmacol Ther 2006;24:1643-9. 